\title{
A framework for user- and system-oriented optimisation of fuel efficiency and traffic flow in Adaptive Cruise Control
}

\author{
Mohammad Mamouei (mohammad.mamouei.1@city.ac.uk) \\ loannis Kaparias \\ George Halikias
}

\begin{abstract}
Fully automated vehicles could have a significant share of the road network traffic in the near future. Several commercial vehicles with full-range Adaptive Cruise Control (ACC) systems or semiautonomous functionalities are already available on the market. Many research studies aim at leveraging the potential of automated driving in order to improve the fuel efficiency of vehicles. However, in the vast majority of those, fuel efficiency is isolated to the driving dynamics between a single follower-leader pair, hence overlooking the complex nature of traffic. Consequently fuel efficiency and the efficient use of the roadway capacity are framed as conflicting objectives, leading to fuel-economy control models that adopt highly conservative driving styles.
\end{abstract}

This formulation of the problem could be seen as a user-optimal approach, where in spite of delivering savings for individual vehicles, there is the side-effect of the deterioration of traffic flow. An important point that is overlooked is that the inefficient use of roadway capacity gives rise to congested traffic and traffic breakdowns, which in return increases energy costs within the system. The optimisation methods used in these studies entail high computational costs and, therefore, impose a strict constraint on the scope of problem.

In this study, the use of car-following models and the limitation of the search space of optimal strategies to the parameter space of these is proposed. The proposed framework enables performing much more comprehensive optimisations and conducting more extensive tests on the collective impacts of fuel-economy driving strategies. The results show that, as conjectured, a "short-sighted" user-optimal approach is unable to deliver overall fuel efficiency. Conversely, a system-optimal formulation for fuel efficient driving is presented, and it is shown that the objectives of fuel efficiency and traffic flow are in fact not only non-conflicting, but also that they could be viewed as one when the global benefits to the network are considered. 


\section{Introduction}

During the past decade, environmental concerns have placed the energy efficiency of vehicles at the centre of researchers' efforts. Great leaps have been made in this area by employing a wide range of technologies that improve fuel efficiency, such as the use of lighter materials in car manufacturing, the adoption of more aerodynamic designs, and the introduction of techniques such as pulse and gliding. However, an important factor that is somewhat overlooked is the impact of the behaviour of drivers and of driving strategies on fuel consumption. A search of the relevant literature reveals that this could be due to the difficulties associated with the formulation of energy efficiency in the car following regime. The algorithms proposed in this area are sometimes based on simplistic assumptions and usually lack comprehensive investigations on their collective impacts and stability features. This is due to the fact that the proposed models often rely on complex and computationally demanding machine learning and optimal control theory-based methods, which make their use in large-scale simulations impractical.

In this study, a new approach is proposed which makes use of car-following models in optimisation. The use of car-following models as the basis of control has already been addressed in the literature (Kesting, et al., 2010). The incorporation of car-following models in simulation-based optimisation significantly reduces the complexity of the latter, which then allows its application to much more comprehensive scenarios. Additionally, the provision of control on the basis of car-following models benefits from the remarkable advantage of the extensive knowledge that exists on the collective properties of these models through the numerous studies that are available in the literature on aspects such as stability features and traffic flow characteristics.

Two distinct approaches to the question of fuel efficiency are investigated in the present study; 1 . individual vehicles are considered and their fuel consumptions are minimised, and 2. fuel efficiency is considered from a broader, network-level perspective. While much of the studies in the literature revolve around the former, the latter is somewhat overlooked. A comprehensive analysis of the results sheds light on the important and fundamental differences between the driving strategies produced by the two approaches. 
In section 2 a literature review is presented and subsequently the gap in the literature is identified. In section 3 two new approaches for optimisation of fuel consumption are formulated to cope with the shortcomings of the existing approaches. In section 4 the results are presented. Finally, conclusions and future work are presented in section 5 .

\section{Literature review}

Wu et al. (2011) developed an advisory system that minimised fuel consumption in the acceleration phase before reaching desired velocities and the deceleration phase before coming to a standstill. The system was shown to deliver reductions of $12 \%$ to $31 \%$ in fuel consumption and the objective was defined as the minimisation of the cumulative fuel consumption, given by the VT-micro instantaneous fuel consumption model (Ahn, 1998), within the time interval of interest (deceleration/acceleration period). For this purpose the objective function was discretised and the resulting optimisation problem was then solved using the Lagrange Multiplier Method (LMM).

Themann et al. (2015) proposed a control model for Adaptive Cruise Control systems (ACC) that relied on the optimisation of the velocity profile with respect to fuel consumption. This study used Dijkstra's algorithm to find the optimal velocity profile for known road topography. Porsche's Innodrive ACC has also adopted a similar approach, resulting in about $10 \%$ reduction in fuel consumption (Markschläger, et al., 2012). Hellström et al. (2010) developed a fuel-optimal control model for trucks. In this study, prior knowledge of road topography was used in order to optimise fuel consumption and gear-shifting, and the problem was formulated as a dynamic programming optimisation. In all these studies, fuel economy is obtained by producing a smooth velocity performance and avoiding unnecessary accelerations. Kohut et al. (2009) achieve the same objective by adopting a Model Predictive Control (MPC) framework. This study highlights the trade-off between fuel savings and trip time.

The development of optimal fuel economy control models in the car-following regime of driving is a more challenging task due to the highly unpredictable nature of drivers' behaviours. In the study by Li et al. (2008), cars' tracking capabilities and fuel efficiency were considered in the development of ACC, and in order to ensure fuel efficiency, accelerations were penalised in the objective function. The problem was then formulated as an MPC 
optimisation, and the testing of the control model was carried out by considering its performance in an urban driving scenario and a highway driving scenario; fuel savings of $8.8 \%$ and $2 \%$ were obtained in each scenario respectively. Kamal et al. (2013) developed an MPC-based controller for the car-following regime that saved an average of $13 \%$ in fuel consumption in urban driving scenarios. Similar approaches can be found in other studies (Luo, et al., 2015; Zhao, et al., 2017).

Zhang \& Ioannou (2006) designed a Proportional-Integral-Derivative (PID) controller for the car-following regime for trucks. The proposed method reduced fuel consumption by avoiding unnecessary accelerations and braking, and the objective of the controller was set to track the velocity of the preceding vehicle while maintaining a specified range of spacing. A different approach in tackling the problem of fuel efficiency is based on the use of new technologies. The potential of technologies such as hybrid electric powertrains and telematics, providing traffic-related information (Manzie, et al., 2007) and techniques such as pulse and gliding (Li, et al., 2012) in the reduction of fuel consumption has been investigated in the literature.

Considering studies seeking more fuel-efficient driving behaviour, two categories can be defined. The first category includes studies seeking to optimise fuel consumption for simple scenarios, where there are no additional complexities caused by interactions between vehicles. In this case information about roadway topography or the position of traffic signals is used in order to formulate an optimisation problem and obtain the optimal velocity profile (Wu, et al., 2011; Themann, et al., 2015; Markschläger, et al., 2012; Hellström, et al., 2010; Kohut, et al., 2009). The second category, on the other hand, consists of studies targeting driving conditions, where interaction between vehicles is the defining factor in driving behaviour. In these studies often simplistic assumptions are made about the relationship between fuel consumption and acceleration or driving dynamics in order to reduce the complexity of problem. More importantly, due to the computational cost of the methods used and the natural complexity of the car-following regime of driving, these studies often narrow down the scope of the problem to a single pair of follower-leader and, therefore, overlook the potentially negative impacts of their proposed control strategies on traffic flow and fuel consumption within the network (Li, et al., 2008; Kamal, et al., 2013; Luo, et al., 2015; Zhao, et al., 2017). 


\section{Methodology}

The modelling of car-following behaviour has been an active area of research for more than six decades. Simple models that effectively describe the microscopic and macroscopic features of traffic have been developed and have been widely studied. As a result, a good understanding of different aspects of these models, namely their collective features and stability characteristics, has been established over the years. Some of these models are also integrated in the control of partially automated vehicles. In the present study the potential of these models is leveraged in order to develop a control model that is not only efficient with respect to fuel consumption but also takes into account the collective impacts of the control model on traffic flow. Section 3.1 provides an overview of the methodology of the present study.

\subsection{Overview of the methodology}

The present study includes a number of components that are separately described in the following sections. Figure 1Error! Reference source not found. provides a schematic representation of how these components are used within different stages of this study.

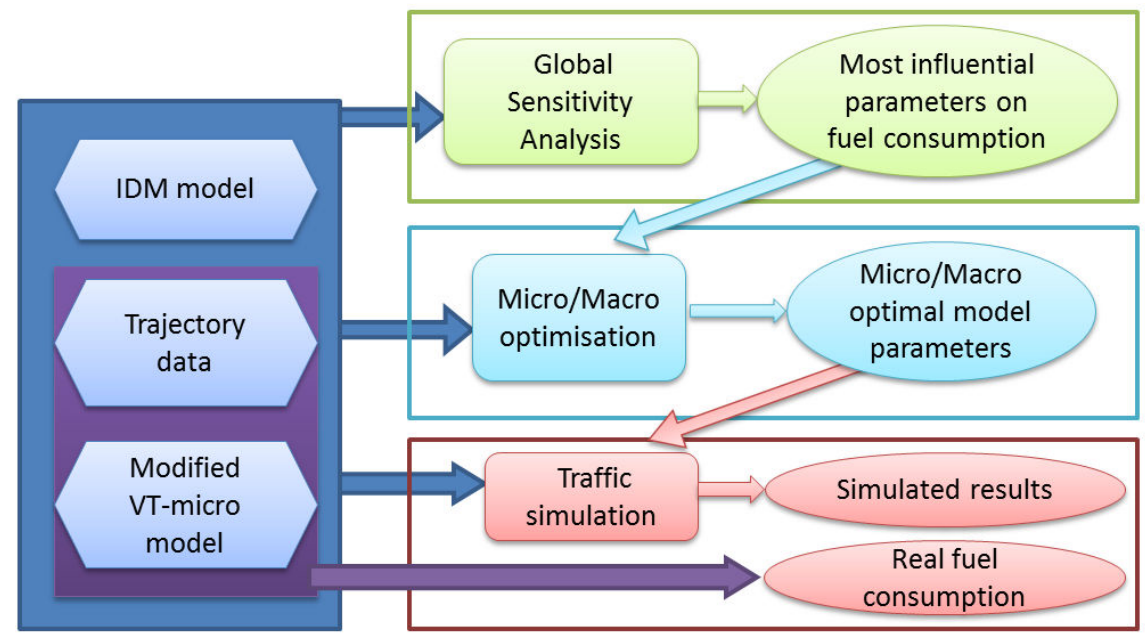

Figure 1 Schematic representation of the present study

Due to the highly nonlinear nature of the problem, a simulation-based optimisation approach is adopted in this study. Two types of simulation scenarios are developed. The first one mainly focuses on the behaviour of a platoon of vehicles that follow a leader, the microscopic features of driving, and the fuel consumption of individual vehicles. The second type mainly focuses on the movement of vehicles within a link, the macroscopic features of driving, and the average fuel consumption within the link. These two types of simulations 
reflect different objectives and lead to drastically different results. The former considers fuel-efficiency from the perspective of individual vehicles (or a platoon of vehicles), while the latter targets fuel efficiency from a wider, link-level perspective. In the former, minimal traffic flow requirements are imposed as constraints on vehicle headway values, which is an important microscopic feature of driving, while in the latter macroscopic measures of flow, such as traffic throughput, can be directly observed and used as constraints. Due to these differences the former is referred to as "microscopic" or "user-optimal" optimisation, while the latter is referred to as "macroscopic" or "system-optimal" optimisation.

In simulation-based optimisation, a simulation scenario may be run thousands of times with different sets of input parameters before the set that minimises the objective function is found. One of the main challenges with this approach is reducing the computational complexity of running the simulations. In the present study, instead of explicitly modelling bottlenecks and sources of disturbance, such as traffic signals and lane changes, which would lead to increased computational requirements, the first vehicle that enters the link (or the leader) is set to follow a given trajectory extracted from a real dataset; the ones used here are the NGSIM-I80 and the Naples datasets. The movement of all other vehicles is modelled using the Intelligent Driving Model (IDM) car-following approach (Treiber, et al., 2000), and estimates of fuel consumption are provided on the basis of the VT-micro model (Ahn, 1998).

This reduction in the computation time comes at the cost of accuracy; nevertheless this approach enables modelling some important dynamic features of traffic and accounting for their impacts on fuel consumption.

The top rectangle on the right-hand-side in Figure 1 corresponds to the sensitivity analysis, whose purpose is to reduce the computation time of the optimisation by focusing only on the parameters of driving that have a major influence on fuel consumption. The rectangle in the middle-right-hand-side depicts the optimisation stage, where the optimal sets of parameters are determined. Finally, the rectangle on the bottom-right-hand-side corresponds to the validation stage. In this stage a number of scenarios are simulated, where vehicles drive according to the optimal model and the fuel consumption values in the 
simulated scenarios are compared with fuel consumption in the corresponding reference case, given by the NGSIM trajectories.

\subsection{The IDM Car-following model}

The IDM car-following approach has been selected for the present study on the basis of its merits. Specifically, the studies available on the macroscopic and microscopic calibration of the IDM point to the good performance of this model on both aspects (Treiber , et al., 2000; Treiber \& Kesting, 2013; Punzo \& Simonelli, 2005). Moreover, the IDM has a simple mathematical form with a small number of parameters, each corresponding to a driving attribute. Finally, numerous studies are available on different aspects of this model, such as calibration, stability and other microscopic and macroscopic properties (Wilson \& Ward, 2011; Kesting \& Treiber, 2009).

The IDM model is given by the following general equation;

$$
\begin{aligned}
& \dot{v}=a\left[1-\left(\frac{v}{v^{d}}\right)^{\delta}-\left(\frac{s^{*}(v, \Delta v)}{s}\right)^{2}\right], \\
& S^{*}(v, \Delta v)=s_{0}+s_{1} \sqrt{\frac{v}{v_{d}}}+T v+\frac{v \Delta v}{2 \sqrt{a b}},
\end{aligned}
$$

$\Delta v=v-v_{p}$,

where, $a$ is the maximum acceleration, $v_{d}$ is the desired speed, $\delta$ is the acceleration exponent, $s_{0}$ and $s_{1}$ determine the jam distances in fully stopped traffic and in high densities respectively, $T$ is the safe time headway, and $b$ is the comfortable deceleration. The input variables are the speed of the subject vehicle, $v$, the speed of the preceding vehicle, $v_{p}$, and the distance headway, $s$. Finally, the output variable, $\dot{v}$, determines the acceleration of the subject vehicle.

\subsection{Optimisation framework}

The coefficients of car-following models can vary according to driving strategies. Given a sufficient number of model parameters, one can reproduce a variety of driving strategies simply by changing the value of model parameters. For example, one set of parameters can represent a sporty driving style with intense accelerations and braking, while another set of model parameters can deliver a more conservative driving style that is less sensitive to the lead vehicle's braking by maintaining sufficient spacing. In other words, a car-following 
model with $\mathbb{N}$ model parameters provides a $\mathcal{R}^{\mathbb{N}}$ space, where each point in this space may be regarded as a distinctive driving style. Therefore, one can seek to find the set of model parameters within this space that minimises an objective function of choice. In this light, calibration studies may be regarded as attempts to find the representations of particular driving behaviour, given by particular trajectories, within the space of model parameters of car-following models.

It is important to note that the space of strategies that is represented by the space of model parameters of a given car-following model is only a subspace of all possible driving strategies. Constraining the search space to the space of model parameters of a carfollowing model has two direct consequences, an advantage and a disadvantage. On the one hand this approach limits the search space to a space where important criteria such as safety, stability, and producing acceptable driving behaviour could be satisfied. This significantly reduces the complexity of the optimisation and mitigates the so-called "curse of dimensionality" that is often encountered in dynamic programming-based approaches. On the other hand, car-following models have shortcomings. A single set of model parameters cannot always produce a realistic driving behaviour. Similarly, one cannot expect a single set of model parameters to always provide a fuel-efficient driving strategy in all driving conditions. Nevertheless, similar to calibration studies, one can obtain model parameters that can deliver fuel efficiency in particular driving conditions.

In this study, two new and distinctive approaches in achieving fuel efficiency relying on the selected car-following model, are investigated: a) an optimisation approach for fuel consumption for a pair of vehicles, or a platoon of vehicles, subject to microscopic constraints on the vehicle headways so as to ensure an efficient traffic flow and adequate tracking capabilities; and b) an optimisation approach of the total fuel consumption within a link, subject to a direct constraint on the traffic throughput. Due to the highly nonlinear formulation of the problem, simulation-based optimisation is carried out to investigate the two approaches.

\subsubsection{Microscopically formulated optimisation}

First the problem of a single pair of follower-leader is considered. The objective is to find the optimal model parameters of the IDM that minimise fuel consumption. For this purpose the 
problem is formulated as follows. Given a particular trajectory of the leader, $T_{l}$, the objective is to find the model parameters, $\theta$, for the IDM car-following model that result in the minimum fuel consumption for the follower.

$J^{*}\left(T_{l}\right)=\min _{\arg \theta \in \Theta} F\left(\theta \mid T_{l}\right)$,

where, $F$ is the fuel consumption of the follower, calculated by a modified version of the VTmicro model (Ahn, 1998). This model is discussed in section 3.4.

In order to ensure that the results have an acceptable tracking capability and meet flowrelated requirements, a wide range of constraints have been considered based on different criteria suggested in the literature (Zwaneveld \& van Arem, 1997; Bierstedt, et al., 2014; Marsden, et al., 2001). The following constraints have produced good results and are, therefore, selected in this study.

$\overline{T_{h}}<\delta_{1}$

$\operatorname{std}\left(T_{h}\right)<\delta_{2}$

where $T_{h}$ is the time series of headways of the follower, $\overline{T_{h}}$ is the mean value of this time series, and $\delta_{1}$ and $\delta_{2}$ are constants. Since the definition of headway becomes problematic in low velocities, a threshold of $18 \frac{\mathrm{km}}{\mathrm{hr}}$ is considered in the calculation of headways and the application of the constraints above.

The results obtained using Equation (2) have been observed to be highly sensitive to the initial conditions and the trajectory of the leader. Therefore, the optimisation framework has been modified to include a platoon of vehicles that follow the trajectory of the lead vehicle. By doing so, string stability features are also implicitly incorporated within the framework. This modification means that intense breaks resulting in unstable or fluctuating acceleration behaviour by the followers, or even potentially leading to accidents, are penalised.

Even though this change pushes the definition of optimality towards a platoon-optimal solution, it serves the purpose of the present study in the following ways: 
1. It contributes to a more practical solution, as the parameters found produce acceptable acceleration behaviour within a platoon.

2. The parameters are much less sensitive to the leader's trajectory and driving conditions. Therefore, the same parameters can be used for a wide range of driving conditions. The proposed framework can be used to find optimal parameters for different driving conditions. Although this would produce more realistic results and further improve fuel efficiency, in the present study, for simplicity, a more generic approach is adopted.

The optimisation framework in this case is formulated as follows:

$J^{*}\left(T_{l}\right)=\min _{\arg \theta \in \Theta} \sum_{i=1}^{n} F_{i}\left(\theta \mid T_{l}\right)$

subject to,

$\overline{T_{h}^{1}}, \overline{T_{h}^{2}}, \ldots, \overline{T_{h}^{n}}<\delta_{1}$

$\operatorname{std}\left(T_{h}^{1}\right), \operatorname{std}\left(T_{h}^{2}\right), \ldots, \operatorname{std}\left(T_{h}^{i}\right), \operatorname{std}\left(T_{h}^{i}\right)<\delta_{2}$

where $n$ is the number of vehicles in the platoon. Additionally, in order to further improve the robustness of optimisation results, the optimisation framework has been further modified to consider more than one trajectory for the leader.

$J^{*}\left(T_{l_{1,2, \ldots, n}}\right)=\min _{\arg \theta \in \Theta} \sum_{i=1}^{n} F_{i}\left(\theta \mid T_{l_{1}}, T_{l_{2}}, \ldots, T_{l_{m}}\right)$

This optimisation formulation is depicted in Figure 2.

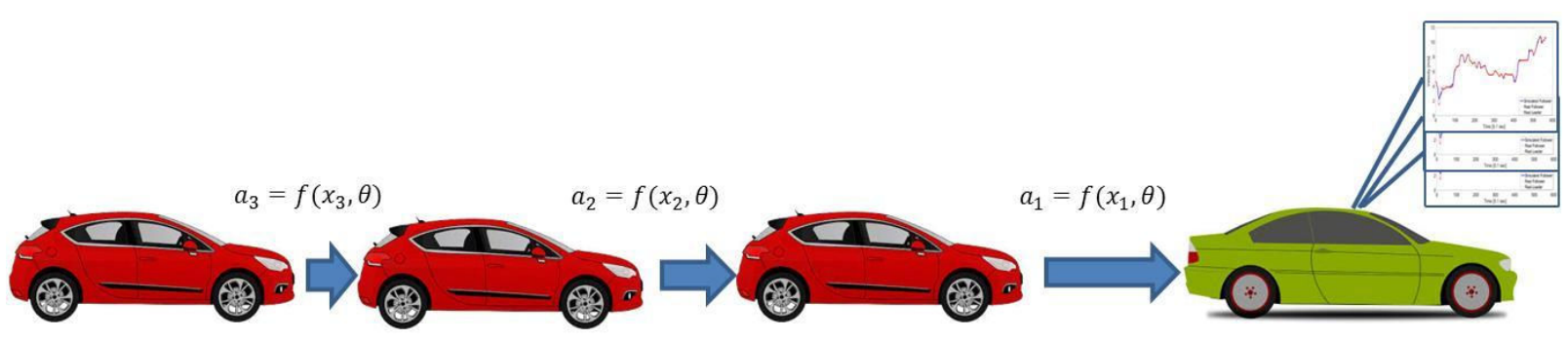

Figure 2 Schematic representation of the microscopic optimisation scenario 
The problem formulation presented in this section is similar to studies related to the carfollowing regime of driving that were addressed in the literature review section. This approach is, in essence, a user-optimal approach in which individual vehicles seek to minimise their individual fuel consumption while respecting certain constraints that ensure an adequate tracking capability and traffic flow.

The approach presented in this section does not address a key aspect of the road network, that is the role of individual entities in forming the collective features of the traffic flow and the mutual impacts that individual agents and their collective behaviour have on one another. The system-optimal optimisation approach presented next captures such usersystem dynamics and mutual impacts.

\subsubsection{Macroscopically formulated optimisation}

For this purpose the horizon of the optimisation problem is broadened in order to,

1. impose macroscopic constraints on the traffic flow, as opposed to the microscopic, headway-based constrains that were previously used, and

2. capture the fuel efficiency properties of the traffic flow in a broader sense than just a platoon of vehicles.

The optimisation problem is modified to the following. Given a particular trajectory, $T_{l}$, for a stretch of a roadway of particular length, $L$, for the simulation time of $t$ seconds, and given the inflow rate of $\lambda$, the objective is to find the model parameters, $\theta$, of the IDM carfollowing model that result in the minimum fuel consumption within the link. This is shown in Equation (6).

$J^{*}\left(T_{l}, \lambda\right)=\min _{\arg \theta \in \Theta} E\left[\sum_{i=1}^{n} F_{i}\left(\theta \mid L, T_{l}, \lambda, t\right)\right]$,

subject to,

Throughput $>\alpha . \lambda . t$,

where the operator $E[$.$] denotes expected value, F_{i}($.$) is the fuel consumption of the i^{t h}$ vehicle following the lead trajectory $T_{l}$ calculated by the fuel consumption model, $n$ is the 
number of vehicles in the scenario, and $\alpha$ is a coefficient that sets a minimum threshold for the acceptable throughput as a percentage of the expected number of vehicles that enter the scenario, which is equal to $t$. The intervals at which vehicles enter the simulation scenario are modelled with the exponential distribution with the average of $\mu=1 / \lambda$. This optimisation framework is schematically presented in Figure 3.

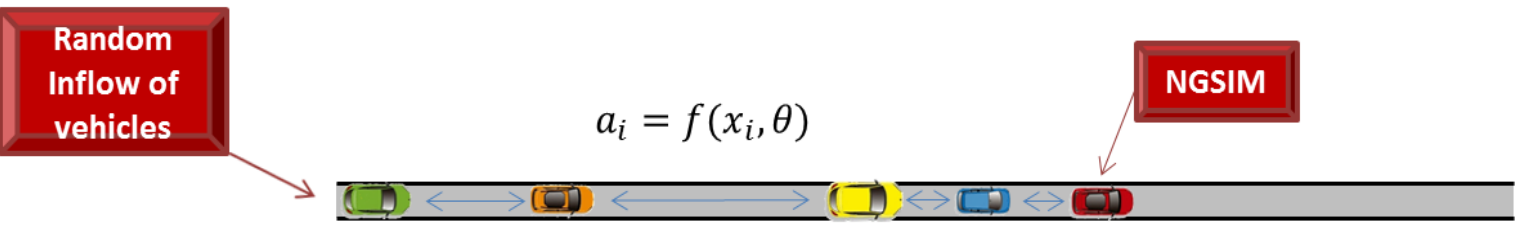

Figure 3 Schematic representation of the macroscopic optimisation scenario.

In Figure 3, the first vehicle that enters the simulated link drives according to a real trajectory extracted from the NGSIM-I80 or Naples datasets. It is worth mentioning that different leader trajectories stimulate different levels of disturbances and shockwaves in the system. Therefore, the shock patterns that are visualised in section 4, depend on the carfollowing behaviour, the inflow of vehicles, and the trajectory of the first vehicle that enters the link.

The appearance of new cars upstream of the link is modelled as a Poisson process. The parameter $\lambda$ of the Poisson distribution determines the inflow rate of vehicles. The driving behaviour of vehicles, apart from the very first vehicle that enters the scenario, is modelled using the IDM car-following model.

In order to highlight the distinction between the macroscopic optimisation scenario and the microscopic one, it should be noted that the macroscopic optimisation scenario is constrained space-wise: this means that vehicles enter and exit the link, but the simulation continues (for a certain period of time), and ultimately the average fuel consumption of all the vehicles is taken into account. On the other hand, in the microscopic simulation there is no explicit representation of a link; instead, a platoon of a predetermined number of 
vehicles, $n$, follow the leading vehicle throughout the simulation. Therein, the main constraint is imposed by the period of time that the leader's trajectory covers.

\subsection{Modified fuel consumption model}

The VT-micro fuel consumption model is specifically developed for investigations related to the operational level of driving, since the only input variables of the model are instantaneous velocity and acceleration (Ahn, 1998). Although the model has a relatively simple structure compared to some other fuel consumption models reviewed in Faris et al. (2011) and Zhou et al. (2016), its dual regime, exponential structure, and large number of terms (16 for each regime), impose a significant computational cost on the optimisation. In order to address this issue a simplified version of the model is developed. The original model and the new simplified model are given by equations (7) and (8), respectively.

$\ln (F(x, y))=\left\{\begin{array}{ll}\sum_{i=0}^{3} \sum_{j=0}^{3} C_{i, j}^{a c c} \times a^{i} \times v^{j} & \text { if } a \geq 0 \\ \sum_{i=0}^{3} \sum_{j=0}^{3} C^{d e c}{ }_{i, j} \times a^{i} \times v^{j} & \text { if } a<0\end{array}\right.$,

$F_{N E W}(x, y)=C_{00}+C_{10} v+C_{01} a+C_{11} v a+C_{02} a^{2}+C_{12} v a^{2}+C_{03} a^{3}$,

where $a$ is acceleration, $v$ is speed, and $C_{i, j}$ are model coefficients.

The new model is validated by comparing its estimates with the original model for the Federal Test Procedure (FTP) drive cycle, which is representative of urban driving. The results are demonstrated in Figure 4. 

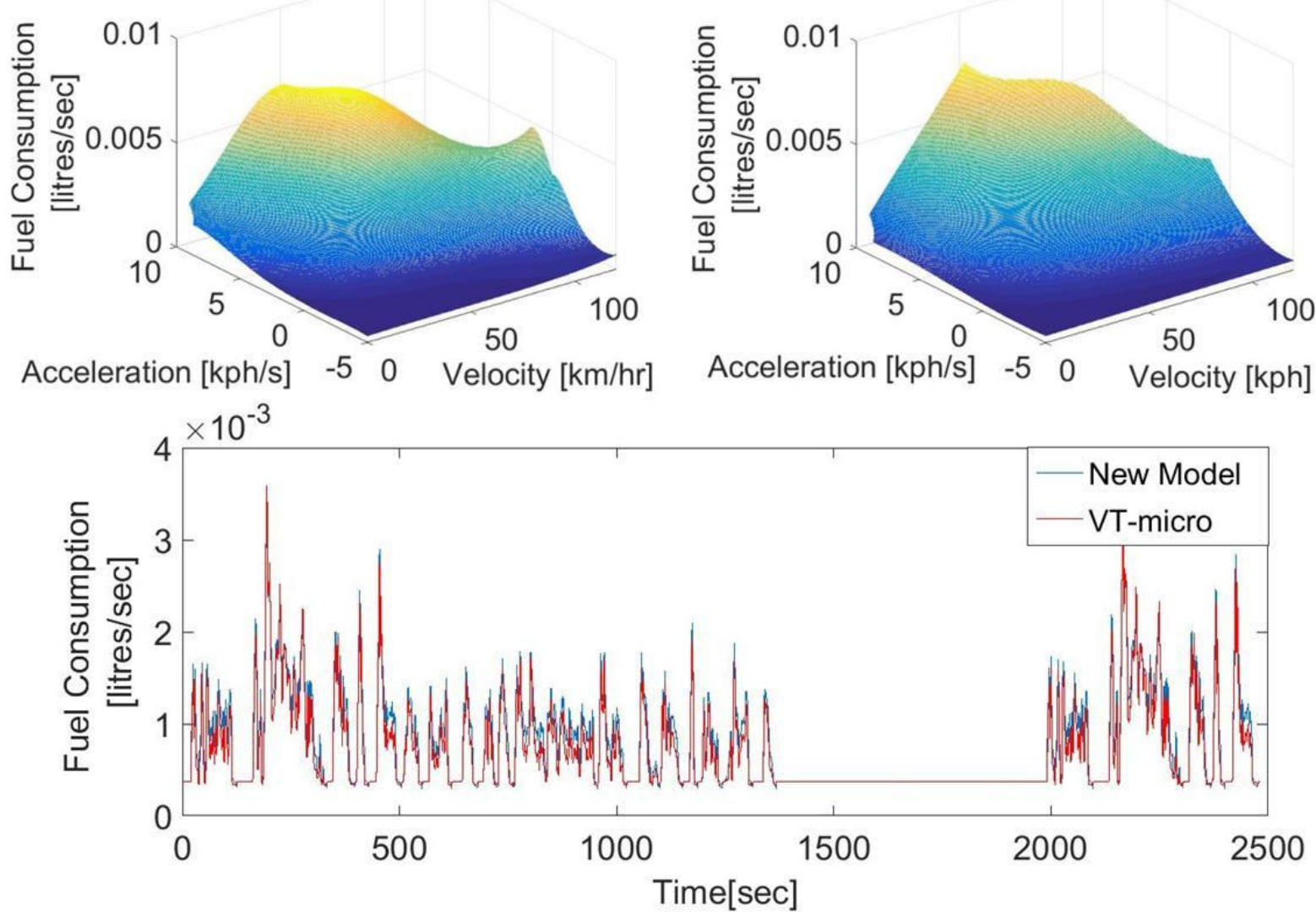

Figure 4 The comparison of fuel consumption prediction between VT-micro and the new model. a, b) Fuel consumption for the whole envelope of velocities and accelerations using VT-micro and the new model respectively. c) Instantaneous fuel consumptions for the FTP drive cycle.

In spite of the much simpler equation of the new model, estimates that are sufficiently close to the original VT-micro model are produced. In particular, the percentage error in total fuel consumption for the FTP drive cycle using the new model is $10.4 \%$, which, for the purposes of fuel-consumption-based optimisation, can be deemed acceptable.

\subsection{Datasets}

Two datasets are used in this study: the reconstructed NGSIM-180 (Montanino \& Punzo, 2015) and the Naples (Punzo, et al., 2005) datasets. The NGSIM-180 dataset provides trajectory data extracted from a stationary camera that covers a 500-meter long stretch of a six-lane highway, which allows the derivation of information about the traffic flow and the overall fuel consumption. Because the original NGSIM-180 dataset contains unrealistically high accelerations which would bias the estimates of fuel consumption, a reconstructed dataset is used in the present study. The Naples dataset, on the other hand, has been obtained by instrumenting four vehicles and measuring their velocities and gaps while they drive through three different routes in Naples (Punzo, et al., 2005). It consists of five 
different sets of data, three of which report spacing and velocity values of the platoon of vehicles while they drive through the three different routes, and the remaining two of which obtained from two previously examined routes on different dates.

Unlike the NGSIM-I80 dataset, the Naples dataset does not provide any information about the surrounding traffic conditions for the subject platoon; however due to the longer period of trajectories for individual vehicles, the absence of lane changes, and the existence of diverse driving conditions, the Naples dataset provides a better insight into car-following behaviour compared to the NGSIM-I80 set. Therefore, in order to make the results of the optimisations relevant to a broader spectrum of car-following conditions, the Naples dataset is used here. The NGSIM-I80 dataset, on the other hand, is used as a reference case for validation purposes. Figure 5 depicts the velocity profiles of the platoon of vehicles in the Naples dataset for trajectories $25 \mathrm{~B}$ and 30B.
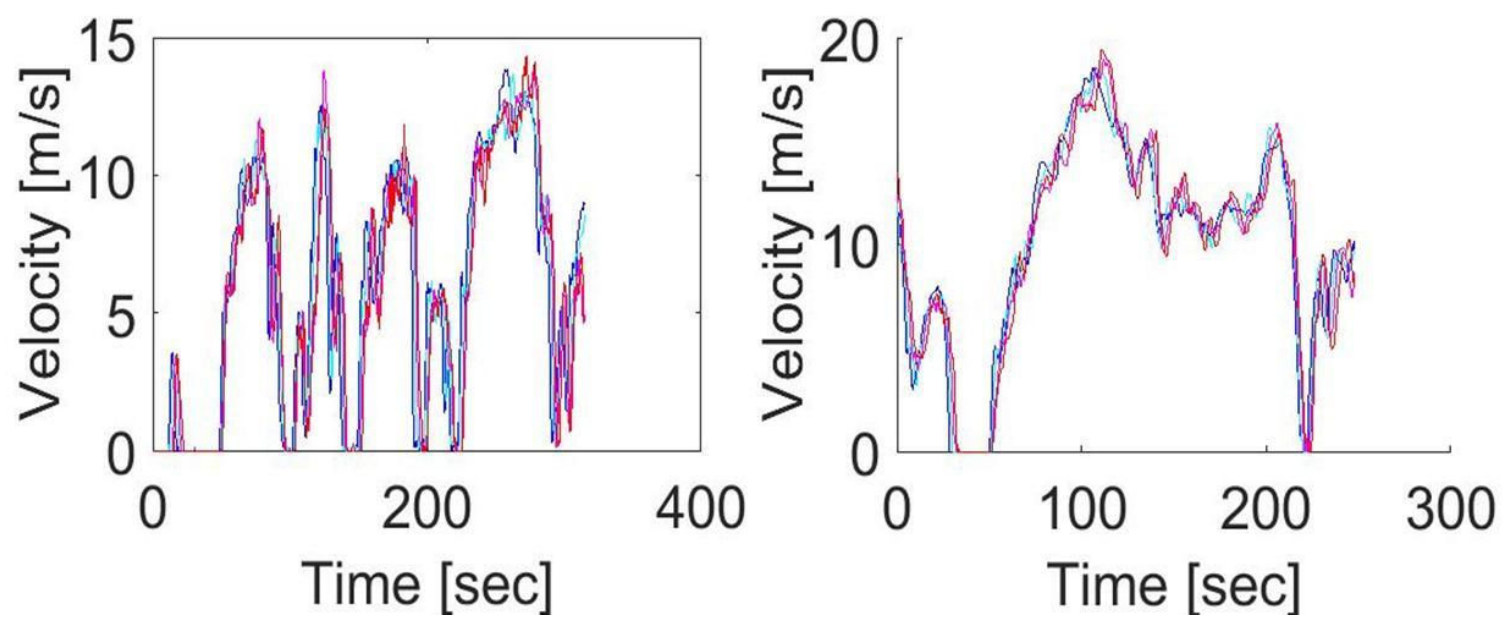

Figure 5 Velocity measurements of the platoon of vehicles in the Naples datasets 25B and 30B.

In order to examine the impacts of the optimal driving strategies in different driving conditions in the validation stage, two trajectories are extracted from lanes 1 and 2 of the NGSIM-I80 dataset. Lane 1 is a High Occupancy Vehicle (HOV) lane and is not disturbed by shockwaves. On the contrary, traffic in lane 2 is congested and numerous shockwaves travelling upstream at a speed of $14 \frac{\mathrm{km}}{\mathrm{hr}}$ can be identified.

The first trajectory used in validation is drawn from lane 1 and relates to the vehicle with ID 441. This vehicle drives with the speed of about $90 \frac{\mathrm{km}}{\mathrm{hr}}$. The second trajectory is extracted from lane 2 and refers to the vehicle with ID 1845. This vehicle drives with an average speed 
of $18 \frac{\mathrm{km}}{\mathrm{hr}}$ and is subject to the main shockwave within the observation period. In the simulations that are presented in section 4 , these trajectories are used as the trajectory of the first vehicle that enters the simulated roadway and the vehicles that follow it drive according to the fuel-consumption-optimised models. The two different trajectories clearly lead to different conditions for the following vehicles; the way in which these conditions affect the flow of vehicles and fuel consumption is examined in section 4.
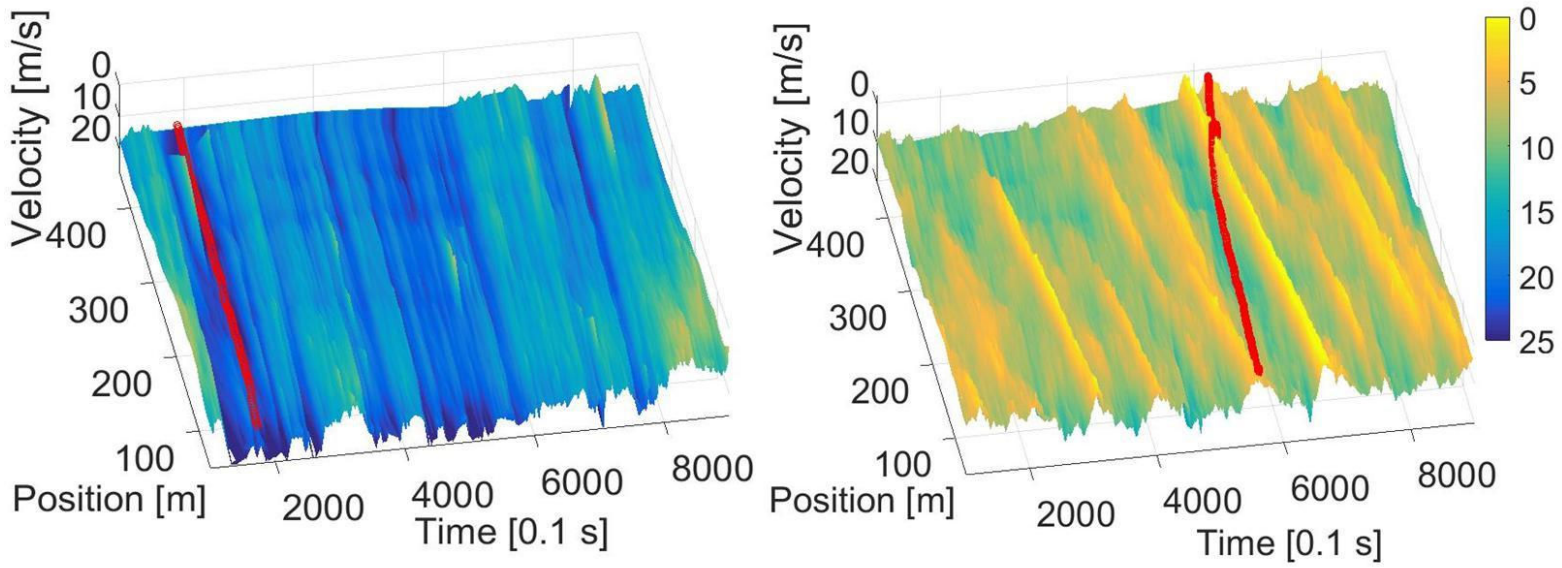

Figure 6 NGSIM-I80 dataset. Trajectories of a) the vehicle with ID 441 from lane 1 and b) the vehicle with ID 1845 from lane 2

The frequent occurrence of lane changes in the NGSIM-180 dataset poses a challenge for the evaluation of fuel consumption within a lane. Hence, fuel consumption results pertaining to the real case are calculated from the vehicles that remain in the subject lane for the whole period of observation. This is depicted in Figure 7.

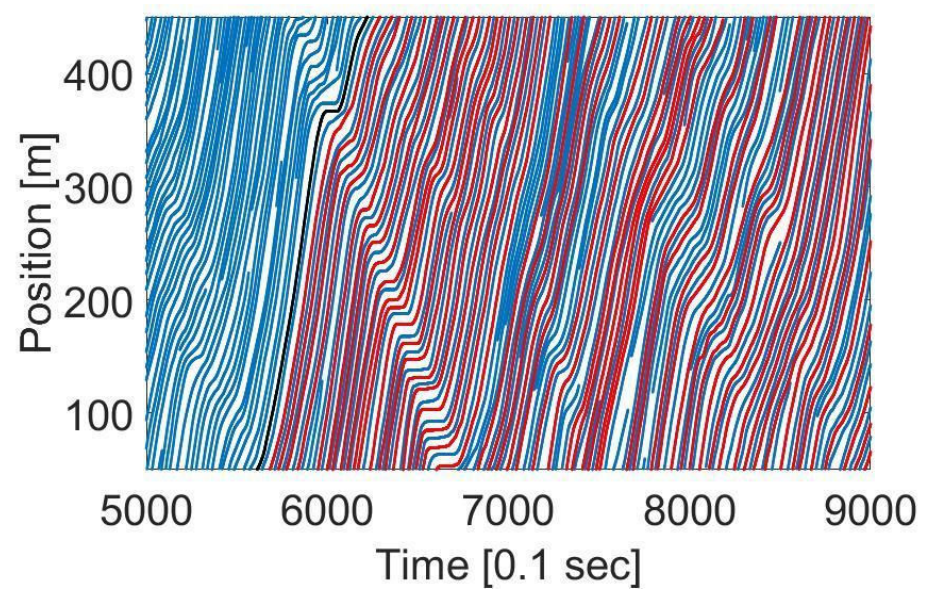

Figure 7 Vehicles that remain in the lane for the whole period of observation are denoted with red 


\subsection{Sensitivity Analysis}

In order to further reduce the complexity of the optimisation a sensitivity analysis is performed to identify the parameters that have the highest impact on fuel consumption. More importantly, without this analysis and by inclusion of parameters with negligible impacts in the calibration, several issues may arise, such as unnecessary computational complexity, overfitting, and potentially misleading results (Punzo, et al., 2015). In this study, the global sensitivity framework is adopted, details of which and of its application to the calibration of car-following models can be found in the literature (Ciuffo, et al., 2014; Saltelli, et al., 2010; Jacques, et al., 2006; Punzo, et al., 2015). The result is illustrated in Figure 8.

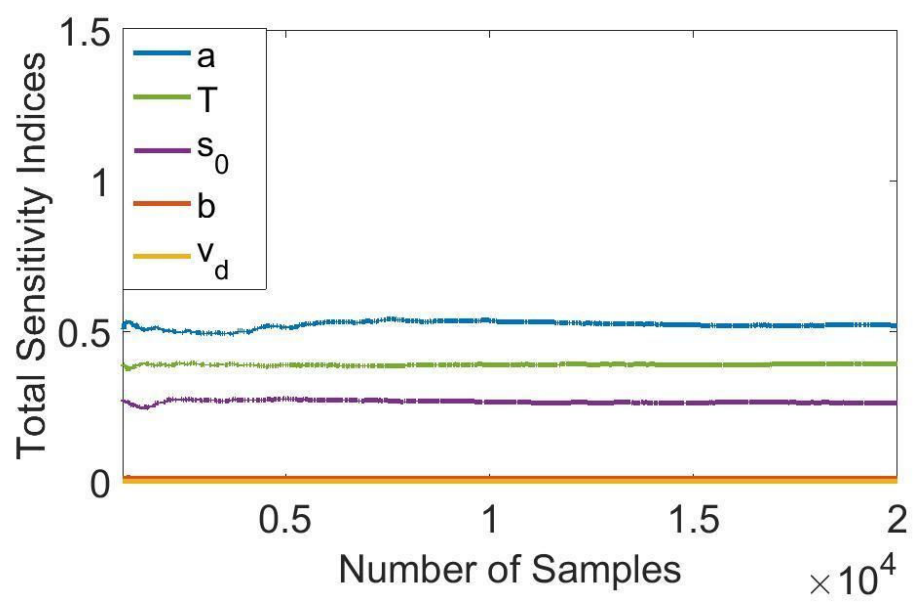

Figure 8 Total sensitivity indices for the effects of the IDM model parameters on fuel consumption in a scenario where the trajectory of the leader is that of the leader in the Naples dataset 25B

It can be seen that parameter $a$ has the highest impact on fuel consumption, followed by parameters $T, s_{0}$ and $b$. Parameter $v_{d}$ has a negligible impact on fuel consumption and is therefore set to its default value of $33.3 \mathrm{~m} / \mathrm{s}$ throughout this study. Parameter $s_{0}$ has a higher impact on fuel consumption compared to parameter $b$. However, for the following reasons the parameter $s_{0}$ is set to its default value of $2 \mathrm{~m}$ :

1. $s_{0}$ represents the jam distance, i.e. a vehicle's desired distance to its leader when both are fully stopped. Therefore, in microscopic calibration this parameter should represent this aspect of driving preference. In macroscopic calibration, however, this parameter plays an important role in determining the shape of the congestion area of the fundamental diagram, especially in high densities and low velocities. The 
calibration of this parameter in the absence of such information can lead to misleading results.

2. $S_{0}$ and $T$ define the desired spacing between vehicles in homogenous driving conditions $(\Delta v=0)$. The two parameters are correlated (Kim \& Mahmassani, 2011) and the impact of $s_{0}$ on spacing at speeds that are considerably greater than zero, can be captured by $T,\left.S^{*}\right|_{\Delta v, s_{1}=0}=S_{0}+v T$.

3. Leaving this parameter out of the optimisation allows to fine-tune it in accordance with other important requirements, such as safety, driver acceptance, and leaving sufficient gaps between vehicles for lane-changes, especially at low speeds.

Although this sensitivity analysis suggests that parameter $b$ has a marginal impact on fuel consumption, this parameter together with $a$ have a strong impact on the stability features of the IDM. Therefore $b$ is also included in the optimisation.

Table 1 demonstrates the lower and upper bound values used in the sensitivity analysis. The same bounds are used for parameters $T, a$ and $b$ in the optimisations. The results of the sensitivity analysis for other datasets are also shown.

Table 1 Lower and upper boundaries used and total sensitivity indices

\begin{tabular}{|c|c|c|c|c|c|c|c|}
\cline { 3 - 8 } & \multicolumn{5}{c|}{ Total sensitivity indices for datasets: } \\
\hline Parameters & LB & UB & 25B & $25 \mathrm{C}$ & $30 \mathrm{~A}$ & $30 \mathrm{~B}$ & $30 \mathrm{C}$ \\
\hline $\boldsymbol{a}$ & 0.5 & 5 & $52 \%$ & $37 \%$ & $85 \%$ & $82 \%$ & $77 \%$ \\
\hline $\boldsymbol{b}$ & 0.5 & 5 & $1 \%$ & $1 \%$ & $2 \%$ & $4 \%$ & $2 \%$ \\
\hline$V_{\boldsymbol{d}}$ & 20 & 33 & $0 \%$ & $2 \%$ & $1 \%$ & $0 \%$ & $0 \%$ \\
\hline$s_{0}$ & 2 & 5 & $26 \%$ & $2 \%$ & $7 \%$ & $17 \%$ & $18 \%$ \\
\hline $\boldsymbol{T}$ & 0.5 & 2 & $39 \%$ & $60 \%$ & $21 \%$ & $14 \%$ & $30 \%$ \\
\hline
\end{tabular}

\section{Results}

\subsection{Microscopically formulated optimisation}

In this section the results relating to the microscopically formulated optimisation problem denoted by Equation (5) are reported. In order to ensure that the results are as robust as possible and deliver fuel efficiency in a wide range of driving conditions, all the trajectories 
available within the five Naples datasets are used as the trajectory of the lead vehicle in the optimisation. Also, the number of vehicles in the platoon is set to 4 , and parameters $\delta_{1}$ and $\delta_{2}$ are set to 3 and 0.5 seconds respectively.

The setting of the values of $\delta_{1}$ and $\delta_{2}$ is a result of a thorough investigation conducted in order to examine the mean and standard deviation of the headways in different driving conditions and for different drivers within the Naples dataset. The mean and standard deviation of headways averaged over the five Naples datasets and all drivers are 1.1 and 0.4 $s$ respectively. These values are highest for the third driver in all five datasets and reach the values of 1.7 and $0.5 \mathrm{~s}$ respectively in dataset 25B. Additionally, numerous combinations of $\delta_{1}$ and $\delta_{2}$ have been tested and it has been confirmed that $\delta_{1}=3 s$ and $\delta_{2}=0.5 s$ produce realistic driving behaviour. The micro-optimal parameters obtained using these parameters and in a scenario where a platoon of three vehicles follow the leader, $n=3$, are $a=$ $1.7\left[\mathrm{~m} / \mathrm{s}^{2}\right], b=1\left[\mathrm{~m} / \mathrm{s}^{2}\right]$, and $T=2[\mathrm{~s}]$.

The optimal set of model parameters results in a $12 \%$ improvement in the fuel consumption of the three following vehicles compared to the total fuel consumption in all five Naples datasets (3.43 litres in the simulated scenario and 3.78 litres in the real case, as estimated by the simplified VT-micro model). The saving is higher in driving conditions where accelerations, stops, and speeding are more dominant. For instance, the reduction in fuel consumption is $13.5 \%$ in dataset $25 \mathrm{~B}$ and $8.2 \%$ in dataset $30 \mathrm{~A}$. 

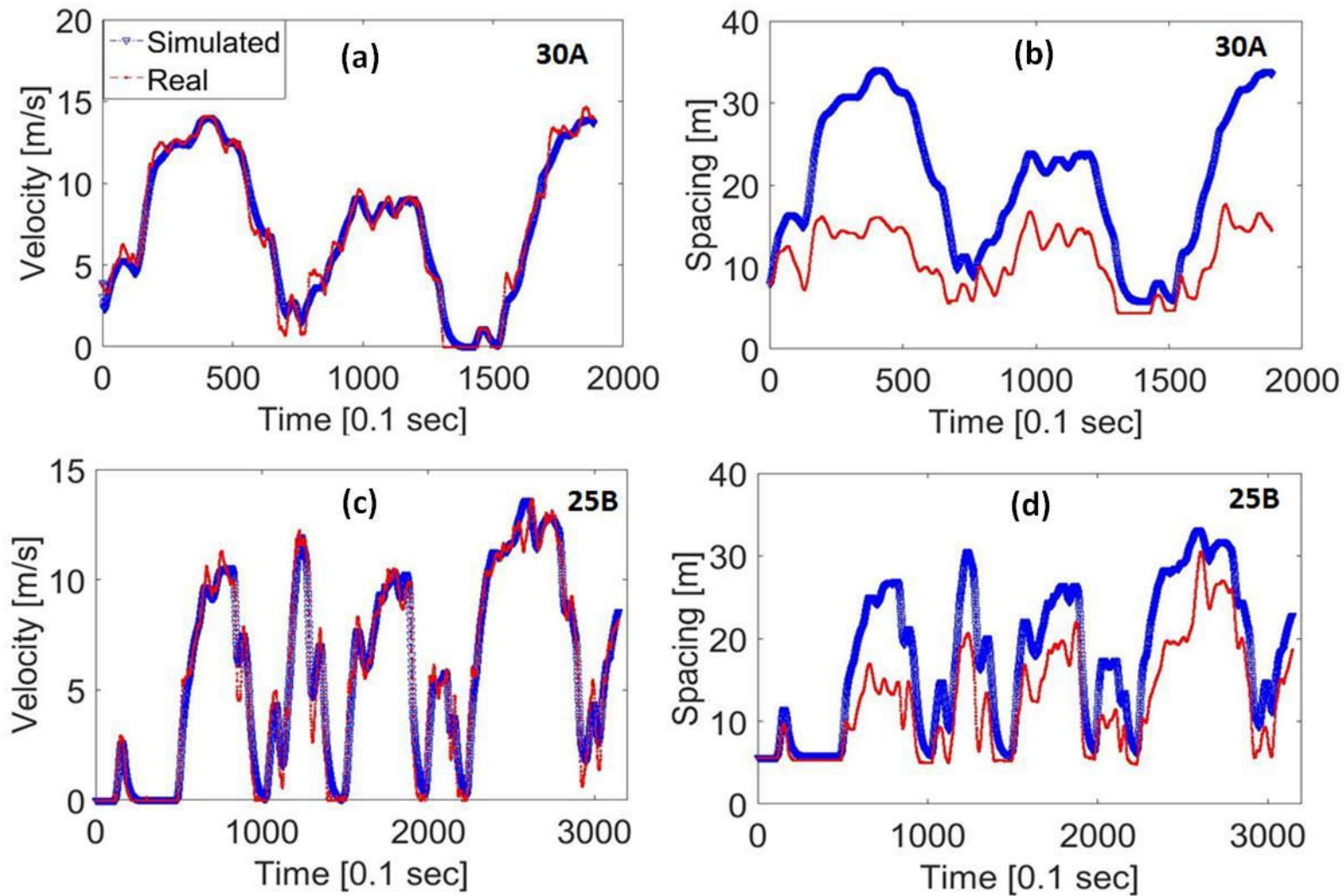

Figure 9 demonstrates the driving behaviour reproduced by the optimal set of model parameters when the trajectory of the lead vehicle is that of the leader in datasets $25 \mathrm{~B}$ and $30 \mathrm{~A}$.
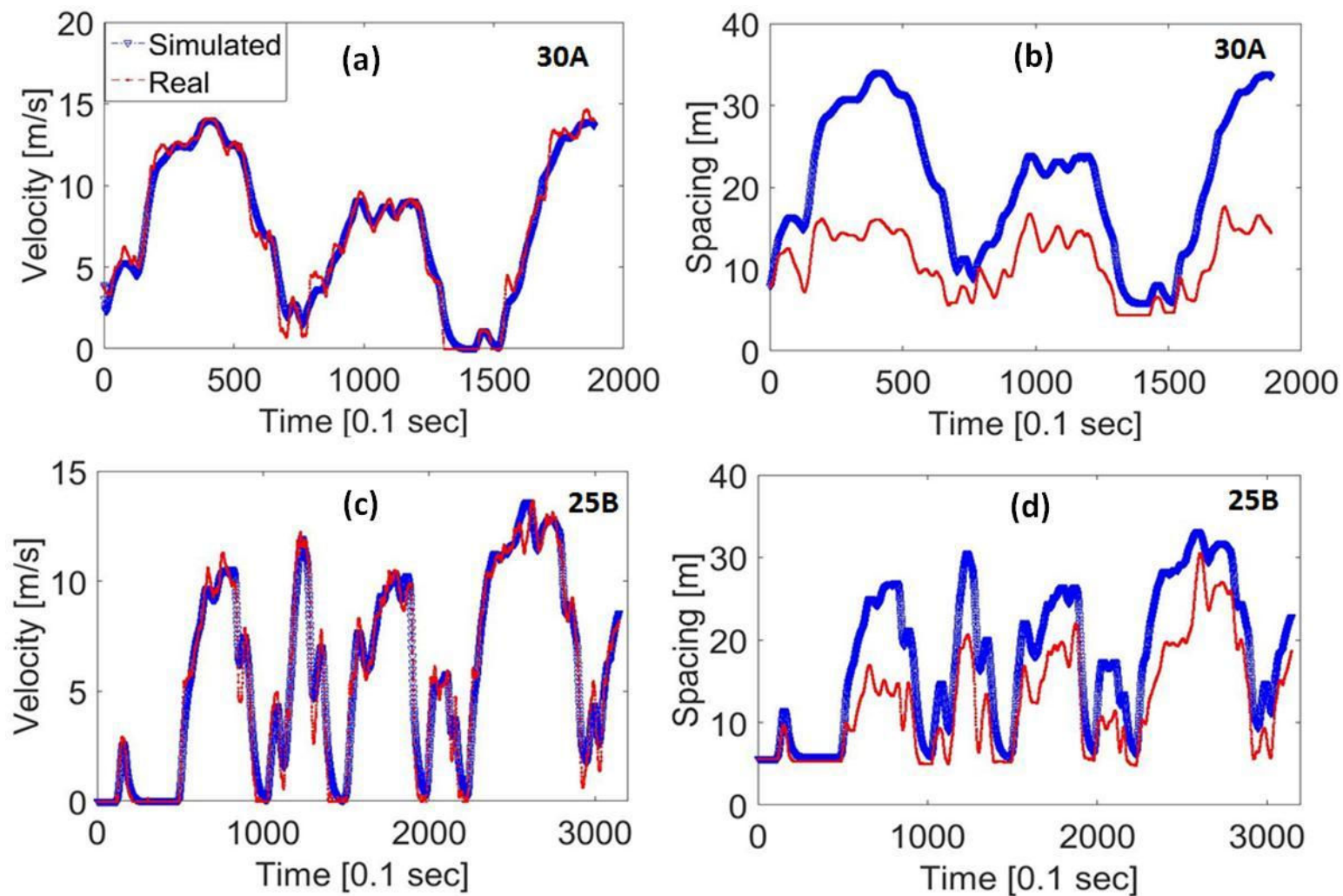

Figure 9 velocity and spacing profiles produced using the optimal parameters (in blue) compared with the real ones (in red) when the trajectory of the lead vehicle is that of the leader in a,b) Naples dataset $30 \mathrm{~A} \mathrm{c,d}$ ) Naples dataset 25B 
It can be seen that the high value of parameter $T$ has resulted in a more conservative driving style, which demonstrates itself through large spacing and a smoother velocity profile. Although such a conservative driving style contributes to the reduction of fuel consumption, it also leads to a reduction of traffic capacity. The deterioration of the traffic capacity means that congested traffic states, intense shockwaves and traffic breakdowns could all occur in lower traffic flows, leading to an increase in fuel consumption for the system. The impact of traffic congestion on fuel consumption was investigated in Treiber et al. (2008) and it was found that traffic congestion could lead to an increase of about $80 \%$ in fuel consumption.

In order to investigate the collective impacts of adopting such driving strategy, a simulation is carried out where the trajectory of the leader is that of vehicle with ID 1845 and the average inflow is set to that of lane 2 between times 540 [s] to 840 [s], which is equal to $1284 v e h / h r$. Figure 10 compares the spatio-temporal velocity profile in the real scenario with the simulated one.
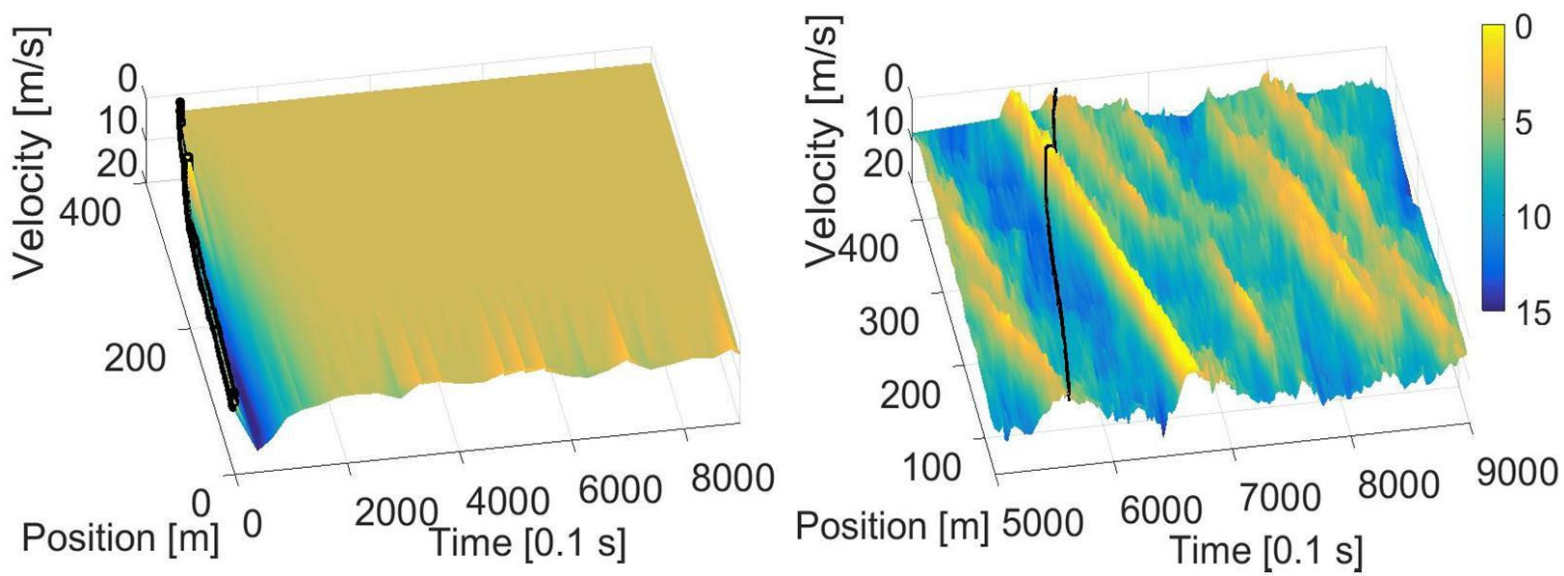

Figure 10 Comparison of the spatiotemporal velocity profile of the modelled scenario with the real data when the trajectory of the lead vehicle is $T_{l}=1845$ (marked with the black solid line)

It can be seen that in the simulated scenario the shockwave triggers a homogenous congested traffic state with an average speed of $14 \frac{\mathrm{km}}{\mathrm{hr}}$. The analysis of the fuel consumption in both scenarios shows that the user-optimal parameters, in spite of delivering fuel savings for the immediate followers, lead to an increase in the average fuel consumption within the link. In particular, the average fuel consumption for the 10 followers of the vehicle with ID 1845 in the real data is $10.5 \frac{\text { litres }}{100 \mathrm{~km}}$. In the simulated dataset this value is reduced to 9.5 $\frac{\text { litres }}{100 \mathrm{~km}}$ (average value in 100 simulations). However, the average fuel consumption for all 
vehicles that follow the vehicle with ID 1845 is $10.3 \frac{\text { litres }}{100 \mathrm{~km}}$ in the real scenario, while for the simulated dataset this is equal to $13.8 \frac{\text { litres }}{100 \mathrm{~km}}$ litres, i.e. a $34 \%$ increase in fuel consumption.

It is important to highlight the impact of the values of $\delta_{1}$ and $\delta_{2}$ on the optimal values. A relaxation of these constraints leads to optimal parameters that have the following values: the lower bound for parameter $a$, the upper bound for parameter $b$, and the upper bound for parameter $T$. While this combination of these values produces more savings for the immediate followers, they result in a much more sluggish driving behaviour. Parameter $T$ determines the headway and parameters $a$ and $b$ together define how agile the following driver's response is to changes in the velocity of the leader. High values of $a$ and low values of $b$ produce a highly reactive driving behaviour, while low values of $a$ and high values of $b$ produce a driving behaviour that is much less sensitive to changes of the velocity of the leader. A sluggish response to changes is beneficial for the fuel consumption of individual vehicles.

The optimal parameters are also largely affected by the trajectory of the leader in the optimisation. For instance, speeding and stops are much more present in dataset 25B compared to dataset $30 \mathrm{~A}$, and the transition from stops to higher speeds occurs faster in dataset $25 \mathrm{~B}$. The selection of trajectories from dataset $25 \mathrm{~B}$ in the optimisation leads to the optimal parameters $a=5 \frac{\mathrm{m}}{\mathrm{s}^{2}}, b=3.2 \frac{\mathrm{m}}{\mathrm{s}^{2}}$, and $T=2 \mathrm{~s}$, while the selection of dataset 30A leads to $a=1.4 \frac{\mathrm{m}}{\mathrm{s}^{2}}, b=0.9 \frac{\mathrm{m}}{\mathrm{s}^{2}}$, and $T=2 \mathrm{~s}$.

As discussed, less sensitivity to changes of the velocity of leader generally contributes to more fuel savings for individual vehicles. However, the optimal parameters for dataset 25B $\left(a=5 \frac{m}{s^{2}}, b=3.2 \frac{m}{s^{2}}\right)$ produce more agile driving compared to the optimal parameters for all five datasets $\left(a=1.7 \frac{\mathrm{m}}{\mathrm{s}^{2}}, b=1 \frac{\mathrm{m}}{\mathrm{s}^{2}}\right)$. In order to address this contradiction, an analysis has been conducted on the acceleration profiles produced by the two sets of parameters. This analysis has revealed that in a driving condition in which changes in the velocity of the leader occur frequently, such as dataset 25B, a less agile driving style produces accelerations of smaller magnitudes. However, this also means that longer periods of acceleration/deceleration must be performed to reach the desired speed, and this could 
lead to an increase in the fuel consumption. Similar results, i.e. $a=4.3 \frac{\mathrm{m}}{\mathrm{s}^{2}}, b=3 \frac{\mathrm{m}}{\mathrm{s}^{2}}$ and $T=2 s$, are found when the number of vehicles in the simulated platoon is increased to $n=10$.

Performing the optimisation using a number of trajectories from lane 1 of the NGSIM-I80 dataset, namely the trajectories belonging to vehicles with IDs $441,452,453$, and 467, confirms this finding. This set of trajectories belongs to a platoon of four vehicles that have a steady speed during the observation period. The optimal parameters found in this case are: $a=0.5 \frac{m}{s^{2}}, b=5 \frac{m}{s^{2}}$, and $T=2 s$.

Microscopic simulation-based optimisation as described above, naturally, has its potential shortcomings. Namely:

1. The optimal values obtained, while producing savings for the immediate followers, could be detrimental to traffic flow and thereby lead to an increase in fuel consumption within the network. This is demonstrated in Figure 11, where a comparison is carried out on the impacts of the model parameters on the flow. In one case the micro-optimal parameters for the case of $n=3\left(a=1.7 \frac{\mathrm{m}}{\mathrm{s}^{2}}, b=1 \frac{\mathrm{m}}{\mathrm{s}^{2}}, T=2 \mathrm{~s}\right)$ are used, and in the second case the calibration results reported in Punzo \& Simonelli (2007) are used. The use of the calibrated model parameters from Punzo \& Simonelli (2007) is particularly interesting since in that study the same dataset (Naples dataset) was used for the calibration of the model parameters.
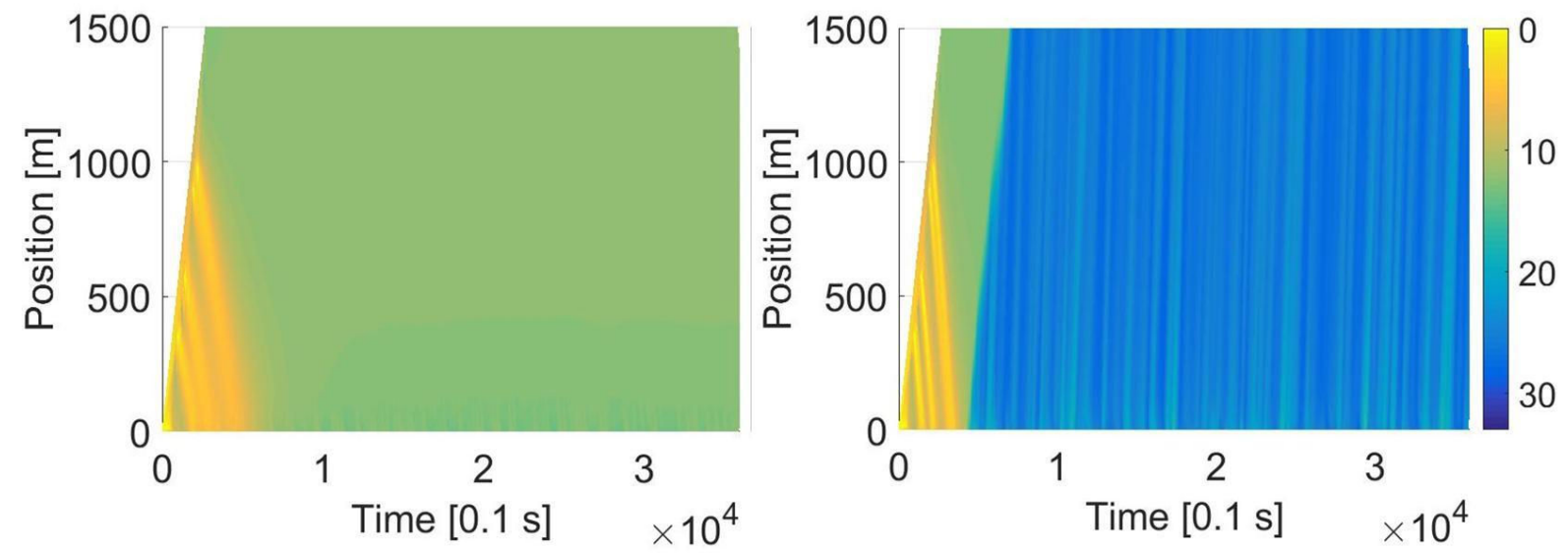

Figure 11 One-hour-long simulation with the inflow of $1800 \frac{\mathrm{veh}}{\mathrm{hr}}$. The trajectory of the leader is that of the leader in the Naples-25B dataset. a) The user-optimal parameters $a=1.7, b=1, T=2$ the rest of the parameters are set to their 
default values, $v_{d}=33.3, \delta=4, s_{0}=2, s_{1}=0$ b) The calibrated values from Punzo \& Simonelli (2007), $a=2.6$, $b=1.7, v_{d}=28.4, \delta=2.84, s_{0}=0.7, s_{1}=0.6, T=0.7$

The results for the two scenarios are summarised in Table 2.

Table 2 The comparison of the micro optimal results with the calibrated set of model parameters from Punzo \& Simonelli (2007)

\begin{tabular}{|l|c|c|}
\hline & $\begin{array}{c}\text { Micro optimal } \\
\text { parameters when } n=3\end{array}$ & $\begin{array}{c}\text { Calibrated parameters } \\
\text { (Punzo \& Simonelli, 2005) }\end{array}$ \\
\hline AFC $^{1}$ within the scenario $\left[\frac{\text { lit }}{\text { vehc.100 } \mathrm{km}}\right]$ & 8.12 & 7.18 \\
\hline AFC for 10 immediate followers & 10.14 & 11.09 \\
\hline Traffic throughput $\left[\frac{\mathrm{veh}}{\mathrm{hr}}\right]$ & 1298 & 1764 \\
\hline
\end{tabular}

${ }^{1} \mathrm{AFC}=$ Average Fuel Consumption

2. Similarly to calibration studies, this type of optimisation is highly sensitive to the driving condition and the set of trajectories used within the optimisation (Punzo, et al., 2015).

It is worth mentioning that the evaluation of fuel consumption based on car-following models that are purely calibrated with respect to individual trajectories or traffic may not produce accurate results for individual vehicles. This subject is thoroughly discussed in Vieira da Rocha et al. (2015). However, the same study concludes that fuel consumption estimates are more reliable when the aggregate fuel consumption for platoons or the total traffic is considered.

The results obtained in this section show that a user-optimal fuel economy driving strategy can be characterised by high headway values. The relaxation of the headway requirements yields the lower bound value for the acceleration parameter and the upper bound value for the deceleration parameter. These parameters have the combined effect of maintaining large spacing between the vehicles in order to afford less sensitivity to minor brake/accelerations of the lead vehicle, hence producing smoother acceleration behaviour. In terms of stability, low values of the parameter $a$ and high values of the parameter $b$ lead to more instability, however, this is compensated by the increase in the value of the parameter $T$ (Treiber \& Kesting, 2011).

It is clear that increased values for parameter $T$ reduce the capacity of the roadway however, this has a negligible impact on the traffic flow when the inflow of vehicles is low and traffic is not in congested states. In other words, an increase in parameter $T$ does not 
change the shape of the left branch of the fundamental diagram for the IDM car-following model. However, as seen above, user-optimal optimisation remains oblivious to the broader perspective of traffic flow and the strategies obtained using such narrowly-framed optimisation formulations can lead to congested traffic states and traffic breakdowns, which in return increase the cost of the trip in terms of fuel consumption within the network.

In what follows, a system-optimal formulation of the problem is presented, where a direct constraint is placed on traffic throughput and the total fuel consumption within the system is the subject of the minimisation.

\subsection{Macroscopic optimisation}

The objective of this section is to broaden the horizon of the optimisation scenario in order to:

1. impose macroscopic constraints on the traffic flow characteristics, as opposed to the microscopic headway-based constraints that were previously used;

2. capture the fuel efficiency properties of the traffic flow in a broader sense than just a platoon of vehicles; and

3. reduce the sensitivity of the optimal parameters to driving conditions.

For this purpose the optimisation framework described in sub-section 3.3.2 is applied here. The input values of the optimisation scenarios are given in Table 3.

Table 3 Features of the simulation scenarios used in the macroscopically formulated optimisations

\begin{tabular}{|c|l|}
\hline$L[\mathrm{~km}]$ & $\begin{array}{l}1.2-2 \mathrm{~km} \text { according to the trip length in the Naples } \\
\text { datasets. }\end{array}$ \\
\hline$t[$ mins $]$ & 5 and 60 minutes. \\
\hline$\lambda=1 / \mu\left[\frac{\text { vehc}}{\mathrm{hr}}\right]$ & $\begin{array}{l}\text { Different values within the range [1080 2664] and } \\
\text { with increments of 200. }\end{array}$ \\
\hline$T_{l}$ & $\begin{array}{l}\text { trajectories from the datasets 25B, 25C, 30A, 30B, } \\
\text { and 30C, from the Naples dataset. }\end{array}$ \\
\hline$\alpha$ & $70 \%$ \\
\hline
\end{tabular}


Three simulation parameters are changed in the optimisation scenario in order to investigate their impact on the optimal parameters: the inflow of vehicles, the trajectory of the lead vehicle, and the simulation time. The variation of the inflow and the lead trajectory allows investigating how robust the optimal parameters are to changes of traffic conditions. The variation of the simulation time enables an assessment of how the consideration of fuel efficiency as both a short-term and a long-term objective affects the optimal strategy.

The optimisation is carried out using a genetic algorithm. Running the optimisation takes between one to three days on a High-Performance Computing (HPC) cluster. Each cluster node consists of two $2.5 \mathrm{Ghz}$ Intel Xeon E5-2670v2 processors, with 40 processors dedicated to the task.

For the short simulation time of $t=5 \mathrm{~min}$ the following optimal values are obtained: $\alpha=0.5, b=5, T=2$. Given the loose throughput requirement and the short timeframe, these values are understandably similar to the user-optimal results with relaxed headway requirements.

When the simulation time is extended to one hour the following parameters are obtained: $\alpha=5, b=0.5, T=0.5$. Conversely to what was previously observed, the lower bound value for headway is obtained, which ensures an increase in the capacity of traffic flow. The upper bound value for the acceleration parameter and the lower bound value for the deceleration parameter compensate the instability that arises from the low value of $T$. Interestingly the results are almost completely robust to changes of the leader's trajectory, variation of the inflow, and even further relaxation of the throughput requirement by reducing $\alpha$ to $50 \%$ (small variations in the value of parameter $b$ take place when the inflow of vehicles goes above $2200 \frac{v e h}{h r}$ ). These results are somewhat counterintuitive, as the driving behaviour produced using these parameters is highly agile, and this contradicts the assumptions made in many studies.

Figure 12 demonstrates the comparison of the system-optimal parameters with the useroptimal ones in a simulation where the trajectory of the first vehicle that enters the scenarios is that of the leader in the Naples dataset 25B and the inflow of vehicles is 1800 $\frac{v e h}{h r}$. The flow-density diagrams are obtained by placing three virtual detectors at $500 \mathrm{~m}$ 
intervals and using the equations below to calculate the average flow and density. The derivation of the fundamental diagram for homogenous traffic is discussed in Treiber et al. (2000).

$Q_{i}=\frac{N_{i}}{T}, \quad \bar{V}_{i}=\frac{N_{i}}{\sum_{j=1}^{N_{i}} \frac{1}{v_{j}}}, \quad \rho_{i}=\frac{Q_{i}}{\bar{V}_{i}}$,

where $T$ is the time interval equal to $1 \mathrm{~min}, N_{i}$ is the vehicle count at time interval $i, v_{j}$ is the velocity of vehicle $j$ that crosses the detector during interval $i, \bar{V}_{i}$ is the harmonic mean of velocities $\left(v_{j}\right), Q_{i}$ is the flow, and $\rho_{i}$ is the density. Each virtual detector counts the number of vehicle crossings, $N_{i}$, and stores their velocities, $v_{j}$. The values of $Q_{i}, \bar{V}_{i}$ and $\rho_{i}$ are then calculated according to Equation (8).
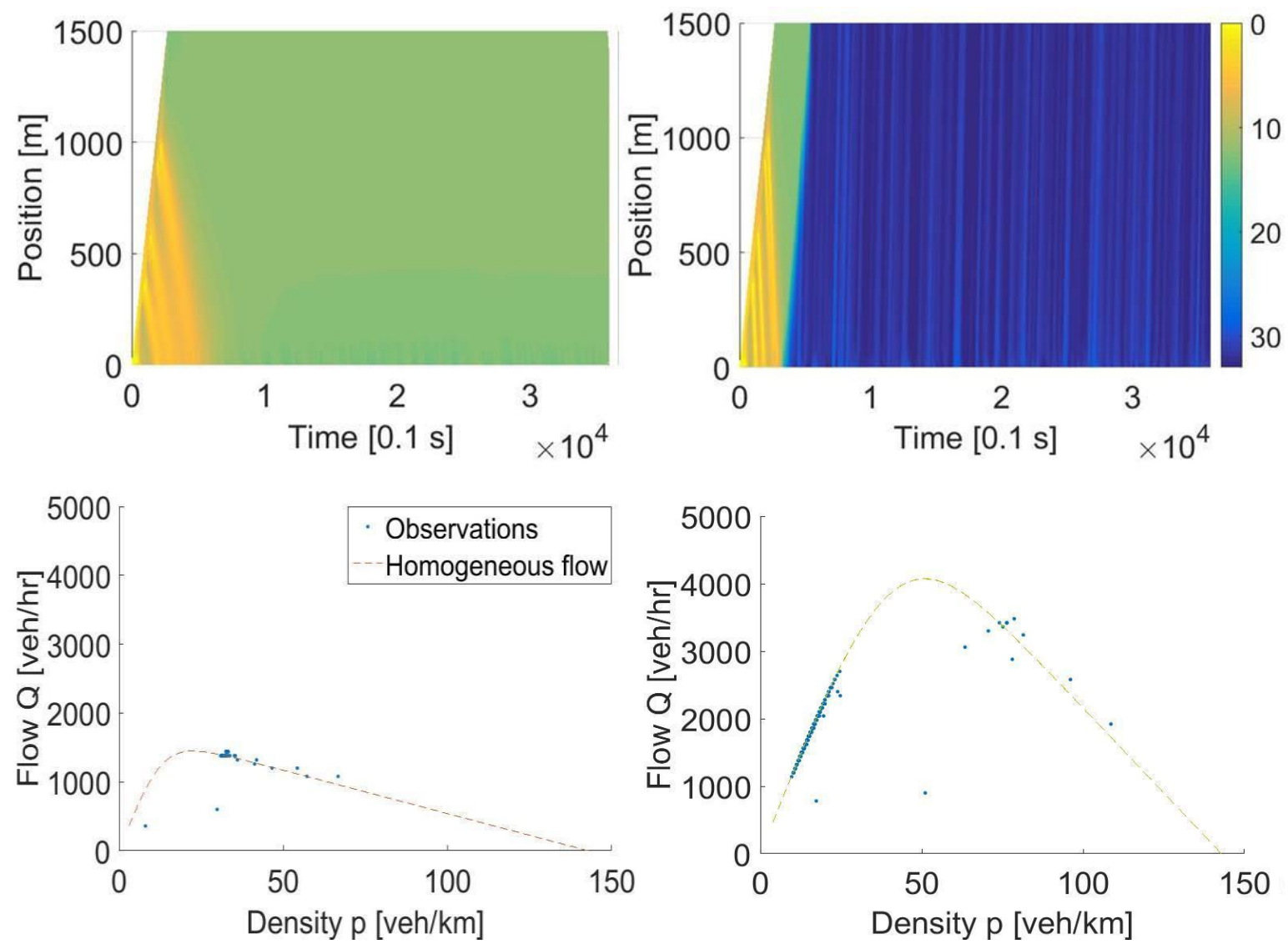

Figure 12 Spatio-temporal graph obtained in a two-hour-long simulation when the trajectory of the first vehicle that
enters the scenario is that of the leader in the Naples dataset 25B. The two figures on the left-hand side correspond to
the user-optimal parameters and the two figures on the right-hand side correspond to the system-optimal parameters.

The use of the system-optimal model parameters results in an average traffic throughput of about $1800 \frac{\mathrm{veh}}{\mathrm{hr}}$ and an average fuel consumption of about $6.68 \frac{\text { litre }}{\text { veh.100 } \mathrm{km}}$, compared to the 
traffic throughput of $1298 \frac{\mathrm{veh}}{\mathrm{hr}}$ and the average fuel consumption of $8.12 \frac{\text { litre }}{v e h .100 \mathrm{~km}}$ for the user-optimal parameters; this significant saving in the total fuel consumption comes at the cost of a $12 \%$ increase in the fuel consumption of the 10 immediate followers of the first vehicle entering the scenario. These values are obtained in a two-hour-long simulation and are averaged over 100 independent simulations. This highlights the fundamental differences between the two approaches to fuel efficiency.

Finally, Table 4 provides a comprehensive comparison of the results. Different sets of parameters were obtained so far from different optimisation approaches (microscopic and macroscopic) and different simulation configurations. These parameters are tested in onehour-long simulations where the trajectories of the vehicles with IDs 441 and 1845 (from the HOV lane and lane 2 of the NGSIM-180 dataset respectively) are used as the the trajectory of the first vehicle that enters the simulation. The inflow of vehicles is adjusted to the real scenario, that is $1284 \frac{v e h}{h r}$ for the scenario when $T_{l}=I D 1845$ and $1344 \frac{v e h}{h r}$ when $T_{l}=I D 441$. The reported values are averaged over 100 simulation runs. Underneath each column of optimal parameter values, Measures of Performance (MoP) relating to fuel consumption and traffic throughput are reported; two values for each set of parameters, one relating to $T_{l}=I D 1845$ and one relating to $T_{l}=I D 441$.

Table 4 Optimal parameters obtained for different optimisations (microscopic and macroscopic) and validation results.

\begin{tabular}{|c|c|c|c|c|c|c|c|c|c|c|}
\hline \multicolumn{11}{|c|}{ Optimal parameters obtained in each optimisation } \\
\hline & \multicolumn{2}{|c|}{$\begin{array}{c}\text { Micro, } \\
n=3 \\
\delta_{1}=3 \\
\delta_{2}=0.5\end{array}$} & \multicolumn{2}{|c|}{$\begin{array}{c}\text { Micro } \\
n=10 \\
\delta_{1}=3 \\
\delta_{2}=0.5\end{array}$} & \multicolumn{2}{|c|}{$\begin{array}{c}\text { Macro } \\
t=5 \text { mins }\end{array}$} & \multicolumn{2}{|c|}{$\begin{array}{c}\text { Macro } \\
t=1 \text { hour }\end{array}$} & \multicolumn{2}{|c|}{ Real } \\
\hline$a\left[m / s^{2}\right]$ & \multicolumn{2}{|c|}{1.7} & \multicolumn{2}{|c|}{4.3} & \multicolumn{2}{|c|}{0.5} & \multicolumn{2}{|c|}{5} & \multicolumn{2}{|c|}{ NA } \\
\hline$b\left[m / s^{2}\right]$ & \multicolumn{2}{|c|}{1} & \multicolumn{2}{|c|}{3} & \multicolumn{2}{|c|}{5} & \multicolumn{2}{|c|}{0.5} & \multicolumn{2}{|c|}{ NA } \\
\hline$T[s]$ & \multicolumn{2}{|c|}{2} & \multicolumn{2}{|c|}{2} & \multicolumn{2}{|c|}{2} & \multicolumn{2}{|c|}{0.5} & \multicolumn{2}{|c|}{ NA } \\
\hline \multicolumn{11}{|c|}{ Validation results for the optimal parameters reported above ${ }^{1}$} \\
\hline Lead Vehicle ID & 441 & 1845 & 441 & 1845 & 441 & 1845 & 441 & 1845 & 441 & 1845 \\
\hline $\mathrm{L}_{-} \mathrm{AFC}^{2}\left[\frac{\text { lit }}{\text { vehc.100km}}\right]$ & 7.1 & 13.8 & 7.3 & 14.2 & 8.5 & 11.3 & 6.4 & 6.9 & 6.9 & 10.3 \\
\hline AFC (10 followers) & 6.7 & 9.5 & 6.3 & 9.4 & 6 & 11 & 7.6 & 10.1 & 6.3 & 10.5 \\
\hline $\operatorname{TTh}^{2}[$ veh $/ h r]$ & 1344 & 963 & 1344 & 937 & 1135 & 1075 & 1344 & 1284 & 1344 & $N A^{3}$ \\
\hline
\end{tabular}


1 Each set of optimal parameters is tested in two simulation scenarios. In the first simulation scenario the lead Both trajectories are obtained from the NGSIM-180 dataset.

${ }^{2}$ L_AFC=Link Average Fuel Consumption, TTH= Trafic Throughput.

3 Not possible to estimate due to the large number of lane-changes.

This table demonstrates that the microscopically optimal parameters (reported in the first two columns of Table 4), in average, result in the reduction of fuel consumption for the immediate followers in comparison with the estimated fuel consumption in the NGSIM-I80 dataset. This can be seen in the row labelled as "AFC (10 followers)" which reports the average fuel consumption for the 10 immediate followers of the vehicles with IDs 441 and 1845. However, this user-optimal (or platoon-optimal) solution causes a much more significant increase in the average fuel consumption within the link. This can be seen in the row labelled as "L_AFC" (Link Average Fuel consumption). A very similar result is obtained when the macro-optimisation is set up in a way that only the very short-term impacts of driving behaviour on fuel consumption are considered, i.e. when $t=5$ mins. On the contrary, the macro-optimal parameters that consider longer-term impacts of driving behaviour on fuel consumption, i.e. $t=1 \mathrm{hr}$, produce a system-optimal approach which although marginally increases fuel consumption for the immediate followers, leads to remarkable savings within the link.

\section{Conclusion and future work}

In this study, a new framework for conducting user- and system-oriented optimisation related to fuel efficiency in the car-following regime was presented. The framework builds on the extensive literature available on car-following models and limits the search space of optimisation to a sub-space of possible driving strategies that is modelled by a car-following model. Depending on the car-following model, this sub-space could represent a search space where important criteria of driving, such as stability, safety, comfort and driver acceptability, are satisfied. Unlike the dynamic programming-based or optimal-controltheory-based methods that can be seen in the literature, the proposed method allows performing large-scale, scenario-based optimisation, and testing the impacts of optimal strategies on the collective features of traffic flow. In this study the focus was on fuel consumption, while other objectives can also be investigated within this framework. 
Firstly, similarly to numerous studies in the literature, the question of fuel efficiency was formulated as the minimisation of the fuel consumption of a vehicle (or a small number of vehicles) while following the leader. The optimal parameters found using the proposed approach produce a driving behaviour that is consistent with the control models proposed in other studies. The optimal parameters yield a driving behaviour that ensures sufficiently large gaps between the vehicles. Furthermore, this strategy is insensitive to minor accelerations/decelerations of the lead vehicle. It was shown that this approach produces a user-optimal driving strategy that, while delivering savings for the immediate followers, leads to a drastic deterioration of traffic capacity. Consequently, the fuel costs of trips significantly increase within the network.

The relationship between fuel consumption and traffic flow is a complex one. Adopting narrowly framed optimisation frameworks based on a single pair of vehicles cannot adequately capture the complex nature of traffic. In order to address this, a system-optimal optimisation was formulated. The system-optimal driving strategy was shown to vary significantly from the user-optimal one. In particular, the user-optimal driving strategy encourages a sluggish driving style with large gaps between the vehicles, while the systemoptimal driving strategy encourages short headways and a highly agile driving behaviour, leading to traffic flow efficiency, as well as network-wide reductions of fuel consumption.

The key finding of the present study is that the application of the user-optimal control models that are widely seen in the literature may be suitable to free-flow conditions, but for more congested states, as is commonly encountered in urban areas, a system-optimal approach, like the one proposed, appears to be more appropriate. And while the study has thrown some light into the topic of energy-efficient ACC, work in this direction continues. Specifically, the next step of the research entails the consideration of the effects of lane changing and of the heterogeneous nature of traffic, which were not addressed here. These subjects and their impacts on the results will be thoroughly analysed in a future study, where the model will be implemented in a realistic urban network consisting of mixed traffic. Further future research will also investigate the use of other suitable car-following models and the application of the framework to different objectives. 


\section{Acknowledgement}

The paper highly benefited from the valuable comments of the anonymous reviewers. The authors would also like to thank Dr. Vincenzo Punzo and Dr. Marcello Montanino for sharing their remarkable work in the reconstruction of the NGSIM-180 dataset and for providing the Naples dataset.

\section{Funding}

The research reported has been supported by City University London's Doctoral Studentships scheme.

\section{References}

Ahn, K., 1998. Microscopic fuel consumption and emission modeling, Blacksburg: Civil and Environmental Engineering. Virginia Polytechnic Institute and State.

Bierstedt , J., Gooze, A., Gray, C., Peterman, J., Raykin, L., \& Walters, J., 2014. Effects of Next Generation Vehicles on Travel Demand and Highway Capacity.[Online] Available at: http://orfe.princeton.edu/ alaink/Papers/FP NextGenVehicleWhitePaper012414.pdf

Ciuffo, B., Punzo, V. \& Montanino, M., 2014. Global sensitivity analysis techniques to simplify the calibration of traffic simulation models. Methodology and application to the IDM car-following model. IET Intelligent Transport Systems, 8(5), pp. $479-489$.

Faris, W.F., Rakha, H.A., Kafafy, R.I., Idres, M., \& Elmoselhy, S., 2011. Vehicle fuel consumption and emission modelling: an in-depth literature review. Intenational Journal of Vehicle Systems Modelling and Testing, 6(3/4), pp. 318-395.

Hellström, E., Åslund, J. \& Nielsen, L., 2010. Design of an efficient algorithm for fuel-optimal lookahead control. Control Engineering Practice, 18(11), pp. 1318-1327.

Jacques , J., Lavergne, C. \& Devictor, N., 2006. Sensitivity analysis in presence of model uncertainty and correlated inputs. Reliability Engineering \& System Safety, 91(10-11), pp. 1126-1134.

Kim, J. \& Mahmassani, H. S., 2011. Correlated Parameters in Driving Behavior Models: Car-Following Example and Implications for Traffic Microsimulation. Transportation Research Record, Issue 2249, pp. 62-77. 
Kamal, A. S., Mukai, M., Murata, J. \& Kawabe, T., 2013. Model Predictive Control of Vehicles on Urban Roads for Improved Fuel Economy. IEEE Transactions on Control Systems Technology, 21(3), pp. 831-841.

Kesting, A., Treiber, M. \& Helbing, D., 2010. Enhanced Intelligent Driver Model to Access the Impact of Driving Strategies on Traffic Capacity. Philosophical Transactions of the Royal Society A 368, pp. 4585-4605.

Kohut, N. J., Hedrick, J. K. \& Borrelli, F., 2009. Integrating Traffic Data and Model Predictive Control to Improve Fuel Economy. Redondo Beach, CA, USA, Proceedings of the 12th IFAC Symposium on Transportation Systems, 42(15), pp. 155-160.

Li, S. E., Peng , H., Li , K. \& Wang, J., 2012. Minimum Fuel Control Strategy in Automated CarFollowing Scenarios. IEEE Transactions on Vehicular Technology, 61(3), pp. 998-1007.

Li, S., Li, K., Wang, J., Zhang, L., Lian, X., Ukawa, H., \& Bai, D., 2008. MPC based vehicular following control considering both fuel economy and tracking capability. Harbin, China, Proceedings of the Vehicle Power and Propulsion Conference.

Luo, Y., Chen, T., Zhang, S. \& Li, K., 2015. Intelligent Hybrid Electric Vehicle ACC With Coordinated Control of Tracking Ability, Fuel Economy, and Ride Comfort. IEEE Transactions on Intelligent Transportation Systems, 16(4), pp. 2303-2308.

Manzie, C., Watson, H. \& Halgamuge, S., 2007. Fuel economy improvements for urban driving: Hybrid vs. intelligent vehicles. Transportation Research Part C: Emerging Technologies February 2007, 15(1), pp. 1-16.

Markschläger, P., Wahl, H. G., Weberbauer, F. \& Lederer, M., 2012. Assistance System for Higher Fuel Efficiency. ATZ Worldwide, 114(11), pp. 8-13.

Marsden, G., McDonald, . M. \& Brackstone, M., 2001. Towards an understanding of adaptive cruise control. Transportation Research Part C: Emerging Technologies, 9(1), pp. 33-51.

Montanino, M. \& Punzo, V., 2015. Trajectory data reconstruction and simulation-based validation against macroscopic traffic patterns. Transportation Research Part B: Methodological, Volume 80, pp. 82-106.

Punzo, V., Formisano ,. D. J. \& Torrieri, V., 2005. Nonstationary Kalman Filter for Estimation of Accurate and Consistent Car-Following Data. Transportation Research Record, Issue 1934, pp. 3-13. 
Punzo, V., Montanino , M. \& Ciuffo, B., 2015. Do We Really Need to Calibrate All the Parameters? Variance-Based Sensitivity Analysis to Simplify Microscopic Traffic Flow Models. IEEE Transactions on Intelligent Transportation Systems, 16(1), pp. 184 - 193.

Punzo, V. \& Simonelli, F., 2005. Analysis and comparison of microscopic traffic flow models with real traffic microscopic data. Transportation Research Record, 1934(1), pp. 53-63.

Saltelli, A., Annoni, P., Azzini, I., Campolongo, F., Ratto, M., \& Taranto, S., 2010. Variance based sensitivity analysis of model output. Design and estimator for the total sensitivity index. Computer Physics Communications, 181(2), pp. 259-270.

Themann, P., Bock, J. \& Eckstein, L., 2015. Optimisation of energy efficiency based on average driving behaviour and driver's preferences for automated driving. IET Intelligent Transport Systems, 9(1), pp. 50-58.

Themann, P. \& Eckstein, L., 2012. Modular Approach To Energy Efficient Driver Assistance Incorporating Driver Acceptance. IEEE Intelligent Vehicles Symposium, Alcalá de Henares, Spain.

Treiber, M., Hennecke, A. \& Helbing, D., 2000. Congested traffic states in empirical observations and microscopic simulations. Physical Review E, 62(2), pp. 1805-1824.

Treiber, M. \& Kesting, A., 2011. Evidence of convective instability in congested traffic flow: Asystematic empirical and theoretical investigation. Transportation Research Part B: Methodological, 45(9), pp. 1362-1377.

Treiber, M. \& Kesting, A., 2013. Microscopic Calibration and Validation of Car-Following Models - A Systematic Approach. Procedia - Social and Behavioral Sciences, 80(7), pp. 922-939. doi:10.1016/j.sbspro.2013.05.050.

Treiber, M., Kesting, A. \& Thiemann, C., 2008. How Much Does Traffic Congestion Increase Fuel Consumption and Emissions? Applying Fuel Consumption Model to NGSIM Trajectory Data. Proceedings of the 87th annual meeting of the transportation research board, Washington D.C.

Vieira da Rocha, T., Leclercq, L., Montanino, M., Parzani, C., Punzo, V., Ciuffo, B., \& Villegas, D., 2015. Does traffic-related calibration of car-following models provide accurate estimations of vehicle emissions?. Transportation Research Part D: Transport and Environment, Volume 34, pp. 267-280.

Wu, C., Zhao, G. \& Ou, B., 2011. A fuel economy optimization system with applications in vehicles with human drivers and autonomous vehicles. Transportation Research Part D: Transport and Environment, 16(7), pp. 515-524. 
Zhang, J. \& loannou, P., 2006. Longitudinal control of heavy trucks in mixed traffic: environmental and fuel economy considerations. IEEE Transactions on Intelligent Transportation Systems , 7(1), pp. 92-104.

Zhao, R. C., Wong, P. K., Xie, Z. C. \& Zhao, J., 2017. Real-time weighted multi-objective model predictive controller for adaptive cruise control systems. International Journal of Automotive Technology, 18(2), p. 279-292.

Zhou, M., Jin, H. \& Wang, W., 2016. A review of vehicle fuel consumption models to evaluate ecodriving and eco-routing. Transportation Research Part D: Transport and Environment, Volume 49, pp. 203-218.

Zwaneveld, P. \& van Arem, B., 1997. Traffic effects of Automated Vehicle Guidance systems. A literature survey, Delft, Netherlands: Department of Traffic and Transport, Delft University, Report INRO-VVG 1997-17. 